EESTI NSV TEADUSTE AKADEEMIA TOIMETISED. XI KÖIDE

FOOSIKALIS-MATEMAATILISTE JA. TEHNILISTE TEADUSTE SEERIA. 1962, NR. 3

ИЗВЕСТИЯ АКАДЕМИИ НАУК ЭСТОНСКОИ ССР. ТОМ ХІ

СЕРИЯ ФИЗИКО-МАТЕМАТИЧЕСКИХ И ТЕХНИЧЕСКИХ НАУК, 1962, № 3

\title{
МЕТОД ИЗМЕРЕНИЯ РАЗНОСТИ ФАЗ ПРИ ПОМОЩИ СИНХРОННОГО ПОЛЯРИСКОПА
}

\author{
Х. АБЕН, \\ кандидат технических наук
}

Для измерения разности фаз световых колебаний при просвечивании двупреломляющих пластинок или фотоупругих моделей широко используется метод Сенармона [']. Несмотря на обширную литературу, посвященную теории и приложениям метода Сенармона, случай, когда скрешенные между собой поляризатор и анализатор поворачиваемы только синхронно, не нашел пока рассмотрения. Так как полярископы указанного типа находят в практике широкое применение (например, производимые экспериментальными мастерскими НИИММ ЛГУ полярископы КСП), то представляет интерес разработать для синхронного полярископа метод измерения разности фаз, аналогичный методу Сенармона.

Синхронный полярископ (фиг. 1) состоит из источника света (ИС), светофильтра (СФ), скрещенных между собой и поворачиваемых синхронно поляризатора (П) и анализатора (А), и из пластинок в четверть волны (Л), которые можно также исключать из оптической системы. Между пластинками в четверть волны ставится модель (M). Обычно одноименные главные направления пластинок в четверть волны взаимно перпен-

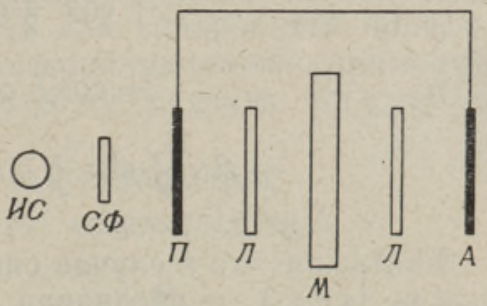

Фиг. 1. дикулярны.

Чтобы измерить разность фаз при помощи синхронного полярископа, нужно определить, при каких условиях интенсивность выходящего из полярископа света равна нулю и каким образом соответствующее положение поляризатора связано с измеряемой разностью фаз.

Длл качественного анализа оптических явлений в синхронном полярископе целесообразно использовать геометрический метод представления поляризованного света [ $\left.{ }^{2}\right]$. Пользуясь геометрическим методом, легко убедиться, что при скрещенных одноименных главных направлениях пластинок в четверть волны интенсивность выходящего из полярископа света равна нулю только тогда, когда разность фаз модели $\delta_{x}$ равна $2 m \pi(m=0,1,2, \ldots)$. Следовательно, такой полярископ не позволяет измерять разности фаз.

Если же поворачивать одну пластинку в четверть волны на $90^{\circ}$, т. е. поставить соответствуюшие главные направления пластинок в четверть 
волны параллельно, то обстановка изменяется. При помоши геометрического метода легко убедиться, что в этом случае всегда сушествует положение поляризатора, при котором интенсивность выходящего из полярископа света равна нулю. Оказывается, что при этом не имеет значения, какой именно является разность фаз пластинок Л. Важно лишь, чтобы их разности фаз были равны между собой. Из этих положений и исходим при разработке метода измерения разности фаз.

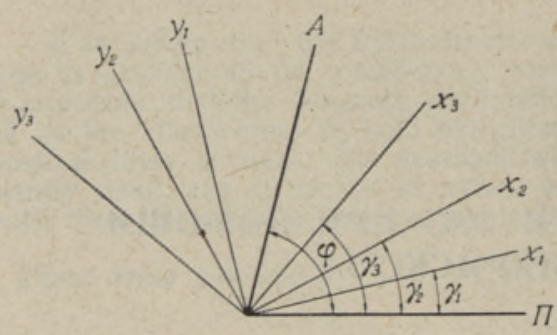

Фиг. 2.

B литературе [3] имеется формула для интенсивности выходящего из анализатора света при просвечивании трех, поставленных друг за другом, двупреломляющих пласлинок. Если обозначить углы между поляризатором и одноименными главными направлениями дв.упреломляющих пластинок $\gamma_{t}$ (фиг. 2), угол между поляризатором и анализатором $\varphi$ и разности фаз двупреломляющих пластинок $\delta_{i}$, то интенсивность выходящего из анализатора света выражается формулой

$$
\begin{aligned}
I=\left[\cos \frac{\delta_{3}}{2} \cos \frac{\delta_{2}}{2} \cos \frac{\delta_{1}}{2} \cos \varphi-\cos \frac{\delta_{3}}{2} \sin \frac{\delta_{2}}{2} \sin \frac{\delta_{1}}{2} \cos \left(\varphi-2 \gamma_{2}+2 \gamma_{1}\right)-\right. \\
-\cos \frac{\delta_{2}}{2} \sin \frac{\delta_{3}}{2} \sin \frac{\delta_{1}}{2} \cos \left(\varphi-2 \gamma_{3}+2 \gamma_{1}\right)- \\
\left.-\cos \frac{\delta_{1}}{2} \sin \frac{\delta_{3}}{2} \sin \frac{\delta_{2}}{2} \cos \left(\varphi-2 \gamma_{3}+2 \gamma_{2}\right)\right]^{2}+ \\
+\left[\cos \frac{\delta_{3}}{2} \cos \frac{\delta_{2}}{2} \sin \frac{\delta_{1}}{2} \cos \left(\varphi-2 \gamma_{1}\right)+\right. \\
+\cos \frac{\delta_{3}}{2} \cos \frac{\delta_{1}}{2} \sin \frac{\delta_{2}}{2} \cos \left(\varphi-2 \gamma_{2}\right)+ \\
\quad+\cos \frac{\delta_{2}}{2} \cos \frac{\delta_{1}}{2} \sin \frac{\delta_{3}}{2} \cos \left(\varphi-2 \gamma_{3}\right)- \\
\left.-\sin \frac{\delta_{3}}{2} \sin \frac{\delta_{2}}{2} \sin \frac{\delta_{1}}{2} \cos \left(\varphi-2 \gamma_{3}+2 \gamma_{2}-2 \gamma_{1}\right)\right]^{2} .
\end{aligned}
$$

Учитывая, что в случае синхронного полярископа $\varphi=90^{\circ}, \delta_{1}=\delta_{3}=\delta$, $\gamma_{1}=\gamma_{3}\left(x_{1}|| x_{3}\right)$, и обозначая $\gamma_{2}-\gamma_{1}=\gamma, \delta_{2}=\delta_{x}$, получим из (1)

$$
\begin{gathered}
I=\left(\sin 2 \gamma_{1} \sin \delta \cos \frac{\delta_{x}}{2}+\cos 2 \gamma_{1} \sin 2 \gamma \sin \frac{\delta_{x}}{2}+\right. \\
\left.+\sin 2 \gamma_{1} \cos 2 \gamma \cos \delta \sin \frac{\delta_{x}}{2}\right)^{2}
\end{gathered}
$$

Из (2) получим, что интенсивность выходящего из полярископа света равна нулю, если выполнено условие

$$
\operatorname{tg} 2 \gamma_{1}=-\frac{\sin 2 \gamma \sin \frac{\delta_{x}}{2}}{\sin \delta \cos \frac{\delta_{x}}{2}+\cos 2 \gamma \cos \delta \sin \frac{\delta_{x}}{2}}
$$

Так как по (3) $\operatorname{tg} 2 \gamma_{1}$ имеет действительные значения при любых значениях $\delta, \delta_{x}$ и $\gamma$, то в случае рассматриваемого полярископа всегда 
сушествуют два перпендикулярных положения поляризатора, при которых интенсивность выходящего из полярископа света равна нулю. Из соотношения (3) разность фаз $\delta_{x}$ выражается формулой

$$
\operatorname{tg} \frac{\delta_{x}}{2}=-\frac{\sin \delta}{\sin 2 \gamma \operatorname{ctg} 2 \gamma_{1}+\frac{1}{\cos 2 \gamma \cos \delta}}
$$

Измерение разности фаз при помощи синхронного полярископа пронсходит следующим образом. Во-первых, определяют главные направления исследуемой пластинки $x$ и $y$ (фиг. 3). Положительное направление отсчета угла $\gamma$ от главного направления фазовых пластинок Л $\left(x_{л}\right) \ldots$ против часовой стрелки. Затем включают пластинки Л (с параллельными главными направлениями) и находят положение поляризатора, при котором интенсивность выходящего из полярископа света минимальна. Положительное направление отсчета угла $\gamma_{1}$ от $x_{\pi}-$ по часовой стрелке. Разность фаз $\delta_{x}$ опреде-

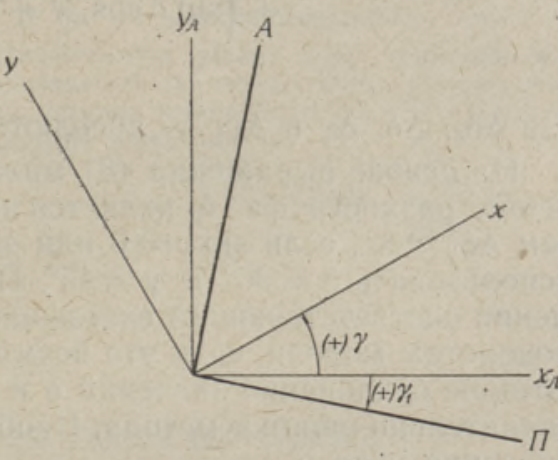

Фиг. 3. ляется по формуле (4). Так как разность фаз определена только при определенной длине волны, то необходимо использовать монохроматический свет.

Как известно, в классическом виде метод Сенармона требует, чтобы фазовая пластинка была пластинкой в четверть волны и ее главные направления составляли бы с главными направлениями исследуемой пластинки угол в $45^{\circ}$. Предложенный метод для синхронного полярископа в этом отношении более сбщий, так как принципиально применим при любых значениях $\delta$ и $\gamma$ (за исключением случаев $\sin \delta=0$ и $\sin 2 \gamma=0$ ).

В некоторых частных случаях формула (4) упрощается.

Пусть $\gamma=45^{\circ}, \delta=90^{\circ}$. Теперь формула (4) принимает вид

$$
\operatorname{tg} \frac{\delta_{x}}{2}=-\operatorname{tg} 2 \varphi_{1}
$$
мона.

В данном случае разработанный метод аналогичен методу Сенар-

Если $\gamma=45^{\circ}, \delta \neq 90^{\circ}$, то имеем из (4)

$$
\operatorname{tg} \frac{\delta_{x}}{2}=-\sin \delta \operatorname{tg} 2 \gamma_{1}
$$

В случае $\gamma \neq 45^{\circ}, \delta=90^{\circ}$ получим из (4)

$$
\operatorname{tg} \frac{\delta_{x}}{2}=-\frac{\operatorname{tg} 2 \gamma_{1}}{\sin 2 \gamma}
$$

Анализируем чувствительность предлагаемого метода к экспериментальным ошибкам. По (4) абсолютная ошибка измеряемой разности фаз выражается в виде 


$$
\begin{gathered}
\Delta \delta_{x}=2 \sin \frac{\delta_{x}}{2}\left(\cos 2 \gamma \sin \frac{\delta_{x}}{2}-\operatorname{ctg} \delta \cos \frac{\delta_{x}}{2}\right) \Delta \delta+ \\
+4\left[\operatorname{ctg} 2 \gamma\left(\sin \frac{\delta_{x}}{2} \cos \frac{\delta_{x}}{2}+\cos 2 \gamma \operatorname{ctg} \delta \sin ^{2} \frac{\delta_{x}}{2}\right)+\right. \\
\left.+\sin 2 \gamma \operatorname{ctg} \delta \sin ^{2} \frac{\delta_{x}}{2}\right] \Delta \gamma+ \\
+\frac{4}{\sin 2 \gamma \sin \delta}\left[\sin ^{2} 2 \gamma \sin ^{2} \frac{\delta_{x}}{2}+\right. \\
\left.+\left(\sin \delta \cos \frac{\delta_{x}}{2}+\cos 2 \gamma \cos \delta \sin \frac{\delta_{x}}{2}\right)^{2}\right] \Delta \gamma_{1}
\end{gathered}
$$

где $\Delta \delta_{x}, \Delta \delta, \Delta \gamma$ и $\Delta \gamma_{1}$ - абсолютные ошибки соответствующих величин.

На основе выражения (8) можно заключить, что ошибка при определении разности фаз $\delta_{x}$ является наименьшей, если $\gamma=45^{\circ}$ и $\delta=90^{\circ}$, причем $\Delta \delta_{x} \rightarrow \infty$, если $\sin \gamma \rightarrow 0$ или $\sin \delta \rightarrow 0$. Следовательно, целесообразно использовать $\delta=90^{\circ}$ и $\gamma=45^{\circ}$. Первое условие легко осуществить, применив соответствующий светофильтр. Второе условие можно выполнить поворотом модели, если это возможно, или поворотом пластинок'Л. Некоторое отклонение значений $\delta$ и $\gamma$ от оптимальных не увеличивает еще значительно ошибки метода. Необходимо лишь избегать слишком малые значения угла $\gamma$. мулой

В случае $\gamma=45^{\circ}, \delta=90^{\circ}$ ошибка определения $\delta_{x}$ выражается фор-

$$
\Delta \delta_{x}=4 \Delta \gamma_{1}
$$

или

$$
\Delta \delta_{x}=\frac{4 \lambda}{360} \Delta \gamma_{1}[\mathrm{MMK}]
$$

где $\lambda$ - длина волны.

Если $\lambda=550$ ммк и $\Delta \gamma_{1}=1^{\circ}$, то имеем

$$
\Delta \delta_{x} \cong 6 \mathrm{Mм \kappa}
$$

Таким образом, в рассматриваемом случае точность метода почти такая же, как у компенсатора Краснова.

В заключение отметим, что для эффективного применения нового метода необходимо точно знать длину волны применяемого светофильтра и соответствующую разность фаз фазовых пластинок.

\section{ЛИ ТЕ Р А Т У Р А}

1. H. de Sénarmont, Sur les modifications que la réflexion spéculaire à la surface des corps métalliques imprime à un rayon de lumière polarisée, Ann. de chim. phys., (2), 73, 1840, p. 337.

2. F. E. W right, A spherical projection chart for use in the study of elliptically polarized light, J. Opt. Soc. Am., 20, 10, 1930.

3. Hsien-Yü Hs ü, M. Richartz, Y üng-K' a ng Li a ng, A generalized intensity formula for a system of retardation plates, J. Opt. Soc. Am., 37, 2, 1947. 


\title{
MEETOD FAASINIHKE MOOTMISEKS SUNKROONPOLARISKOOBI ABIL .
}

\author{
H. Aben, \\ tehniliste teaduste kandidaat
}

\section{Resümee}

Esitatakse Sénarmont' meetodiga analoogiline meetod faasinihke mõõtmiseks sünkroonpolariskoobi (joon. 1) abil. Näidatakse, et juhul, kui «veerandlaine-plaatide» vastavad peasuunad on paralleelsed, eksisteerib alati selline polarisaatori asend, mille puhul polariskoobist väljuva valguse intensiivsus on null. Sellel pōhinebki faasinihke mōõtmine. Otsitav faasinihe $\delta_{x}$ määratakse seosest (4), kus $\delta$ on «veerandlaine-plaatide» faasinihe ( $\delta$ võib erineda $\pi / 2$-st), $\gamma$ on nurk uuritava kaksikmurdva plaadi ning «veerandlaineplaadi» peasuundade vahel ja $\gamma_{1}$ on nurk polarisaatori ja «veerandlaine-plaadi» peasuundade vahel juhul, kui polariskoobist väljuva valguse intensiivsus on null. Esitatud meetodi täpsus ei ole halvem Krasnovi kompensaatori täpsusest.
Eesti NSV Teaduste Akadeemia Küberneetika Instituut
Saabus toimetusse
6. I 1962

\section{A METHOD FOR MEASURING THE PHASE DIFFERENCE BY MEANS OF A SYNCHRONIZED POLARISCOPE}

\section{H. Aben}

\section{Summary}

For measuring the phase difference by means of a synchronized polariscope, a method is presented which is analogous to the method of Senarmont. It is shown that if the principal directions of the "quarter-wave" plates are parallel, there always exists a position of the polarizer, by which the intensity of the emergent light is zero. On this fact the method is based. The unknown phase difference $\delta_{x}$ is determined from the formula (4) where $\delta$ is the phase difference of the "quarter-wave" plates ( $\delta$ can be different from $\pi / 2)$, $\gamma$ is the angle between the principal directions of the "quarter-wave" plates and the plate under investigation, and $\gamma_{1}$ is the angle between polarizer and the principal directions of the "quarter-wave" plate when the emergent light has zero intensity. The precision of the method is the same as by the compensator of Krasnov.

Academy of Sciences of the Estonian S.S.R.,

Received Institute of Cybernetics 